\title{
Research Paper \\ An Evaluation of Infection Types and the Complications in Hospitalized Elders Admitted in ICU Wards
}

\author{
Majid Zare Bidaki ${ }^{1} \odot$, Zohreh Azarkar $^{1} \odot$, Gholamreza Sharifzadeh $^{1} \odot{ }^{*}$ Azadeh Ebrahim Zadeh $^{1} \odot$
}

1. Infectious Diseases Research Center, Birjand University of Medical Sciences, Birjand, Iran.

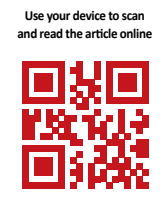

dtation: Zare Bidaki M, Azarkar Z, Sharifzadeh Gh, Ebrahim Zadeh A. [An Evaluation of Infection Types and the Complications in Hospitalized Elders Admitted in ICU Wards (Persian)]. Iranian Journal of Ageing. 2020; 15(1):118-129. https://doi. org/10.32598/sija.15.1.2087.1

doi https://doi.org/10.32598/sija.15.1.2087.1
Key words:

Infection, ICU, Elderly

adults

\section{A B STRACT}

Objectives Infections acquired in ICU wards is a major part of nosocomial infections in each hospital. Controlling this type of infections can reduce mortality and hospitalization time. An important and highrisk target group for these infections involves older adults. This study aimed to investigate the infections acquired in ICU among older adults as well as contributors to and complications of these infections.

Methods \& Materials In this descriptive, analytic study, all the elderly patients ( $>60 \mathrm{y}$ ) hospitalized in two ICUs wards of teaching hospitals of Birjand University of Medical Sciences during 2015 were included. The patients who were being treated by antibiotics or were unwilling to participate or had preliminary positive cultures were excluded. After 72 hours of hospitalization in ICU, a set of participants' samples including blood, urine, wounds, lung discharges, and sputum were cultured. In the positive samples, factors associated with the infection, such as age, sex, initial disease, catheterization, and the complications were examined.

Results In this study, 417 older adults ( $>60 \mathrm{y}$ ) hospitalized in Imam Reza and Vali-e-Asr Hospitals of Birjand with Mean $\pm S D$ age of $74.04 \pm 8.9$ years were entered in the study. Among them, 255 (61.2\%) patients were in Imam Reza Hospital and the remaining were in Vali-e-Asr Hospital. Men were accounted for $45.8 \%$ $(n=191)$ of participants. The infection had a frequency of $18.7 \%(n=78)$. Men showed a higher rate of infection than women ( $24.1 \%$ vs $14.2 \% ; P=0.01$ ). Infections increased significantly as hospitalization time increased (i.e., $2.7 \%$ in patients with $<5$ days, $30.2 \%$ in patients with $5-10$ days; $67.2 \%$ in patients with $>10$ days of hospitalization; $P>0.001)$. The most common infection was respiratory infection observed in $42(53.8 \%)$ of cases followed by urinary infections. There was no significant correlation between age and infection occurrence. Correlation was found neither between infection and mortality, nor between age and mortality. Infection rates was significantly greater in the internal diseases ICU (35.8\%) than in the surgery ICU $(7.8 \%)(\mathrm{P}<0.001)$.

Conclusion Elderly adults hospitalized in the ICU are prone to infections. Thus, the hospitalization time and the intubation times should be reduced as far as possible.

\section{* Corresponding Author:}

Azadeh Ebrahim Zadeh, PhD.

Address: Infectious Diseases Research Center, Birjand University of Medical Sciences, Birjand, Iran.

Tel: +98 (915) 3613525

E-mail:mr14436@yahoo.com 


\section{Extended Abstract}

\section{Introduction}

A

nosocomial infection is a type of infection that occurs 48-72 hours after hospitalization, while the patient is not in the incubation period of the infection at the time of admission [1].

More than $20 \%$ of nosocomial infections occur in the ICU and cause more than $10 \%-80 \%$ deaths [2]. In the elderly, common and classic forms of infectious diseases are rare and appear more atypical and unusual. In a 2016 study by Mancini, 12 of the 19 people with pseudomonas pneumonia in the ICU were over 70 years old [2]. The incidence of many infectious diseases is higher in the elderly than in other age groups, so that the incidence of respiratory and urinary tract infections in the elderly is 20-50 times higher than in the non-elderly [3].

The aim of this study was to investigate the prevalence and risk factors of infections and their complications in the elderly admitted to the ICU of hospitals affiliated to Birjand University of Medical Sciences.

\section{Methods \& Materials}

In this cross-sectional (descriptive-analytical) study, the study population was all elderly patients (over 65 years old) who were admitted to the ICUs of hospitals affiliated to Birjand University of Medical Sciences from April 2015 to March 2016.

ICU of the Valiasr Hospital is an internal ICU, and ICU of the Imam Reza Hospital is a surgical ICU. At the beginning of hospitalization, blood and urine cultures were performed on all patients, and they were cultured with sputum and exudate if they had a sample.

Samples that were initially positive and patients who did not consent to participate in the project or who had taken any antibiotics in the past 72 hours were excluded from the study. The rest of the subjects were sampled again 48-72 hours later. If the test was positive, they remained in the study and those in whom the test result was negative were cultured 48 hours later if they had infection criteria.

Sampling was performed by a trained ICU supervisor under the supervision of an infectious disease specialist. All samples were cultured in a specific medium by a microbiologist. No costs were incurred for patients included in the project and they entered the study with full consent.

\section{Clinical definitions}

In this study, an infection was considered a urinary tract infection in which the person had clinical signs of urinary tract infection and the result of his/her culture was positive and the colony count was $>105 \mathrm{cfu} / \mathrm{ml}$.

Respiratory infection or pneumonia refers to an infection in which a person has a 38-degree fever with cough, purulent sputum, short breaths, pelvic pain, rale sound in the lungs, and a positive radiology for pneumonia. Bacteremia is an infection in which the organism is isolated from blood culture twice without a single site of infection. After collecting data, they were entered into SPSS V. 18 software and analyzed using descriptive statistical tests, frequency percentage and Chi-square analytical test. The code of ethics is IR.bums.REC.1394.17.

Table 1. Comparison of the prevalence of infection in patients admitted to ICU wards by sex and length of hospital stay

\begin{tabular}{|c|c|c|c|c|}
\hline \multirow[b]{2}{*}{ Variable } & \multirow[t]{2}{*}{ Infection } & \multicolumn{2}{|c|}{ No. (\%) } & \multirow{2}{*}{$\begin{array}{c}\text { P } \\
\text { Chi-square Test }\end{array}$} \\
\hline & & Yes & No & \\
\hline \multirow{3}{*}{ Gender } & Male & 46 (24.1) & $145(75.9)$ & \multirow{3}{*}{$=0.01$} \\
\hline & & & & \\
\hline & Female & $32(14.2)$ & $194(85.8)$ & \\
\hline \multirow{3}{*}{$\begin{array}{c}\text { Duration of } \\
\text { hospitalization }\end{array}$} & Less than 5 days & $7(2.7)$ & $257(97.35)$ & \multirow{3}{*}{$<0.001$} \\
\hline & 5-10 days & $26(30.2)$ & $60(69.8)$ & \\
\hline & More than ten days & 45 (67.2) & $22(32.8)$ & \\
\hline
\end{tabular}


Table 2. Comparison of infection incidence in hospitalized patients in ICU based on age and hospital

\begin{tabular}{lcccc}
\hline & \multicolumn{2}{c}{ Infection } & No. (\%) & P \\
\cline { 3 - 4 } & & Positive & Negative & Chi-square test \\
\hline \multirow{2}{*}{ Age } & $60-74$ & $36(17.4)$ & $171(82.6)$ & 0.49 \\
& & $42(20)$ & $168(80)$ & $<0.001$ \\
\multirow{2}{*}{ Hospital } & ICU of Emam Reza & $20(7.8)$ & $235(92.2)$ & $104(64.2)$ \\
\end{tabular}

Table 3. Comparison of death rate based on infection condition in investigated patients

\begin{tabular}{ccc}
\hline & & No. (\%) \\
\cline { 2 - 3 } Infection & Positive & Negative \\
\hline Dead & $24(30.8)$ & $74(21.8)$ \\
Alive & $54(69.2)$ & $265(78.2)$ \\
Total & $78(100)$ & $339(100)$ \\
\hline
\end{tabular}

\section{Results}

This study was performed on 417 elderly people over 60 years of age admitted to the ICU of Imam Reza and Valiasr hospitals in Birjand with a mean age of $74.04 \pm 8.9$ years, a minimum age of 60 years, and a maximum age of 99 years. A total of 255 patients $(61.2 \%)$ were admitted to the ICU of Imam Reza Hospital and the rest were admitted to the ICU of Valiasr Hospital. Also, a total of 191 patients (45.8\%) were male and the rest were female.

The prevalence of infection in the studied patients was $18.7 \%$ (78 patients). The prevalence of infection in males was significantly higher than females $(\mathrm{P}=0.01)$, and the prevalence of infection increased significantly with increasing duration of ICU hospitalization $(\mathrm{P}<0 / 001)$ (Table 1).

The most common infection in the elderly was respiratory infection (53.8\%) and then urinary tract infection. Chisquare test showed that there was no significant statistical difference in the type of infection by age and sex. The prevalence of infection was higher in the elderly over 75 years of age than in people aged 74-60 years, but this difference was not statistically significant. Also, the prevalence of infection in the ICU of Valiasr Hospital was significantly higher than the ICU of Imam Reza Hospital $(\mathrm{P}<0.001)$ (Table 2).

Out of 417 patients studied, 98 people $(23.5 \%)$ died. In patients with positive infection, $30.8 \%$ of 78 people, and in patients with negative infection, $21.8 \%$ of 339 people died. This difference was not statistically significant using Chisquare test $(\mathrm{P}=0.09)$ (Table 3$)$.

In our study, the rate of infection was significantly related to the duration of hospitalization and male gender (Table 1), so that patients with a duration of hospitalization of more than 10 days had $67.3 \%$ infection.

The most common infections in our study were respiratory infections $(53.8 \%)$ and then urinary tract infections (43.1\%), followed by wound infection and bacteremia $(25.5 \%$ and $21.4 \%)$, respectively.

In this study, in terms of pathogenic symptoms, the most common organisms were: Pseudomonas for respiratory infection; E.coli and Pseudomonas and Candida for urinary tract infections; and Staphylococcus aureus and Pseudomonas for wound infection and bacteremia. Also, according to the present study, the prevalence of infections in the ICU of Valiasr Hospital, which is an internal ICU, was significantly higher than the ICU of Imam Reza Hospital, which is a surgical ICU.

In terms of mortality, in our study, although there was no significant relationship between infection and mortality, but quite clearly in people with positive infection mortality was $30.8 \%$ more than people without infection $(21.8 \%)$. 


\section{Conclusion}

In this study, the prevalence of infection was $18.7 \%$. In the above-mentioned ICUs, the infection rate in the elderly was $10.8 \%$.

Various studies have shown that nosocomial infections in the ICUs are 5\%-15\% more than other wards, which can increase to $50 \%$. This may be due to the fact that the clinical signs of infections in the elderly are not typical and this in turn delays in diagnosis and therefore in treatment.

In this study, the rate of infection was significantly related to the duration of hospitalization and male gender (Table 1 ), so that patients with a length of hospital stay of more than 10 days had $67.3 \%$ infection. In the 2014 Solis study, the rate of infection in the ICU was directly related to the length of hospital stay [1].

According to the present study and most studies, the high prevalence of respiratory infections is due to long-term intubations required in the ICU. Therefore, if the elderly are hospitalized in the ICU, it is better to reduce the length of their stay to a minimum.

\section{Ethical Considerations}

Compliance with ethical guidelines

This cross sectional study was approved by the ethics committee of Birjand University of Medical Science (Code: IR.BUMS.REC.1395.107).

\section{Funding}

This study was extracted from a research proposal and received financial support from the Deputy for Research And Technology of Birjand University of Medical Science.

\section{Authors' contributions}

Conceptualization, Investigation: Azadeh Ebrahimzadeh, Majid Zare Bidaki; Methodology: Gholamreza Sharifzadeh; Writing-original draft: Zohreh Azarkar, Majid Zare bidaki; Writing-review \& editing: Majid Zare; Supervision: Azadeh Ebrahimzadeh.

\section{Conflicts of interest}

The authors declared no conflict of interest.
The authors would like to appreciate the ICU supervisors and microbiologists of Vali-e Asr and Emam Reza Hospitals.

\section{Acknowledgements}


This Page Intentionally Left Blank 


\title{
بررسى انواع عفونتها وييامد أنها درسالمندان بسترى در ICU
}

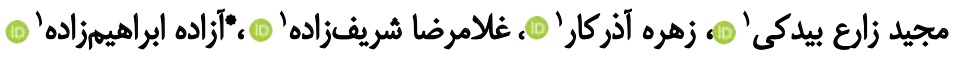

ا. مركز تحقيقات بيمارىهاى عفونى، دانشكاه علوم هزشكى بيرجند، بير جنده ايران.

\begin{abstract}
حكSد

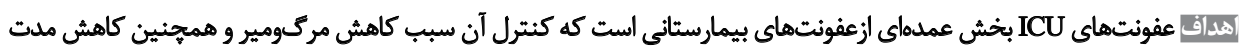

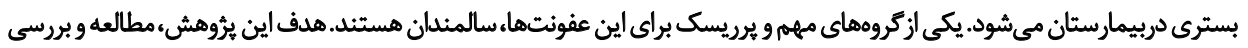

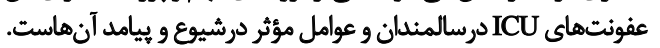

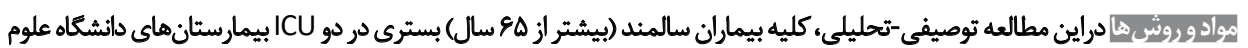

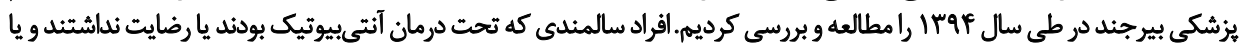

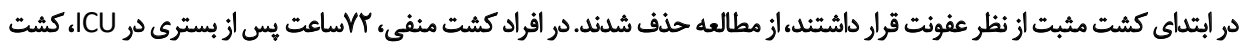

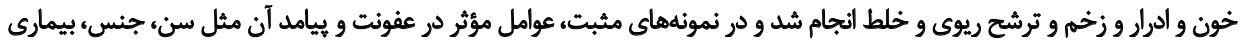

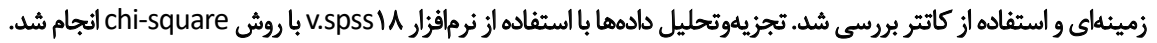

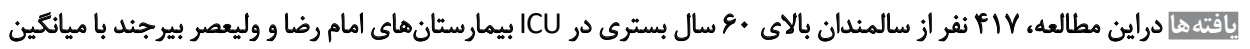

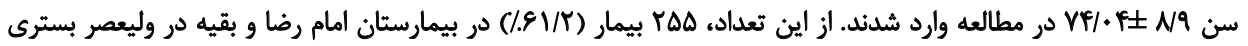

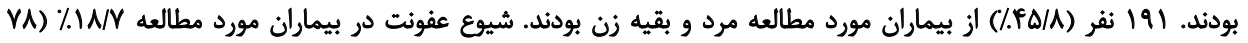

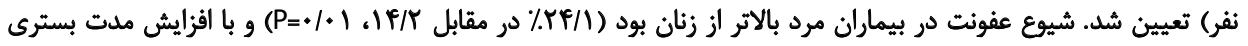

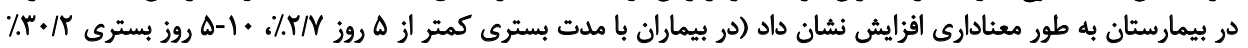

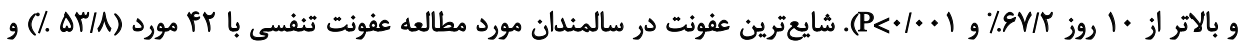

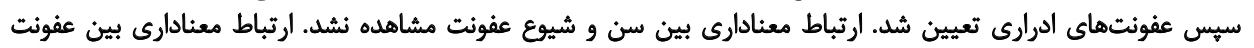

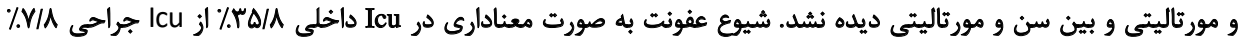

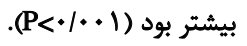
تتيجه كيرى) بيماران سالمند بسترى در ICU مستعد انواع عفونتها هستئد و بايد در حد امكان طول مدت بسترى و انواع لوله كذارى را در آنها كاهش داد.
\end{abstract}

تاريخ دريافت: 1 •اسفند هو

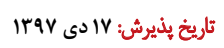

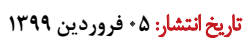

كليدواروها:

عفونتء اله سالمندان

كلبسيلا، يروتئوس و سراشياواز كرم مثبتها، استافيلوكوك طلايى

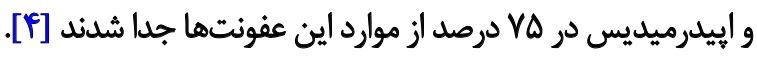

در سالمندان، جهرههاى معمول و كلاسيك بيمارىهاى عفونى

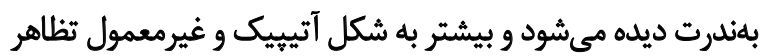

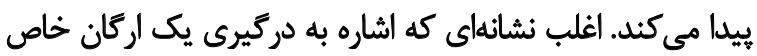

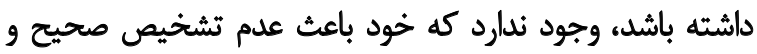

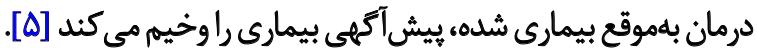

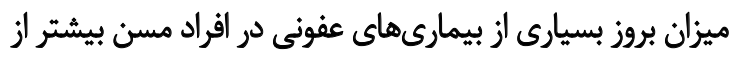

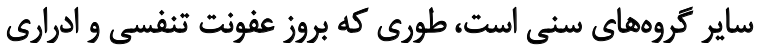

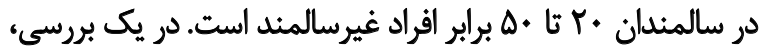

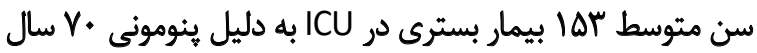

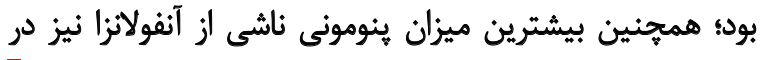

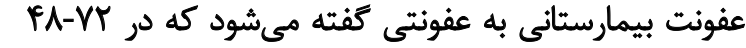

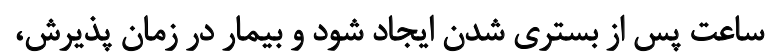

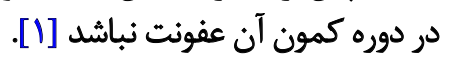

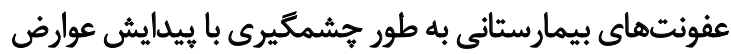

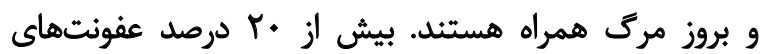

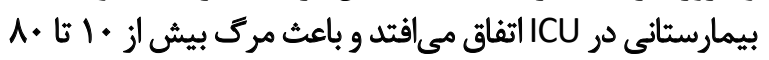

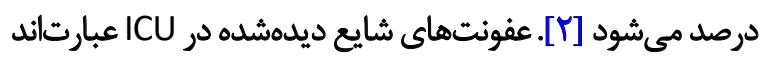

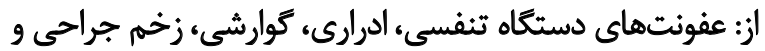

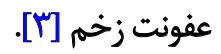
اركانيسمهاى كرم منفى مثل ايشرشياكولاي، بسودومونا،

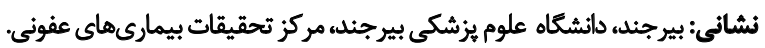
تلفن: T) يست الكترونيكى: mr14436@yahoo.com 


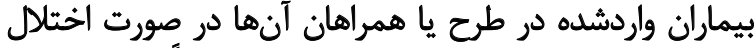

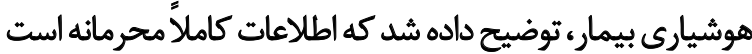

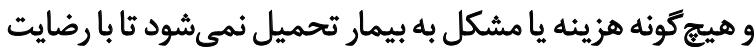
در مطالعه شركت كنيند.

\section{تعاريف بالينى عفونتها بدين ترتيب بود:}

عفونت ادرارى به عفونتى كفته شد كه فرد علائم كلينيكى

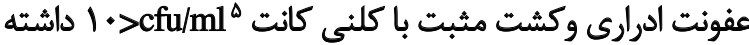

عفونت تنفسى يا پينومونى به عفونتى اطلاق شد كه فرد ثب

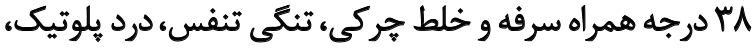

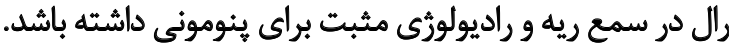

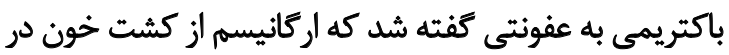

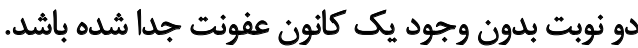

دادهها يس از جمعآورى وارد نرمافزار

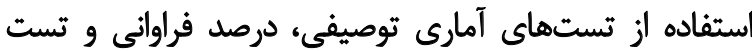

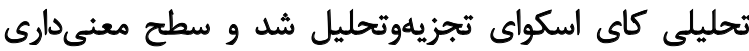

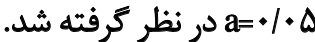

يافتهها

اين مطالعه روى FIV نفر از سالمندان بالاى •\& سال بسترى

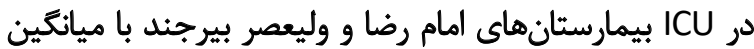

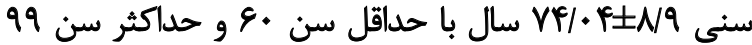

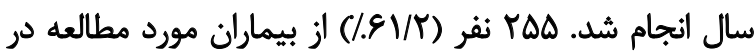

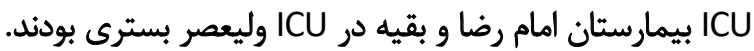

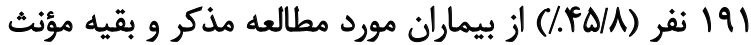

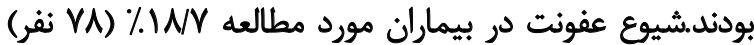

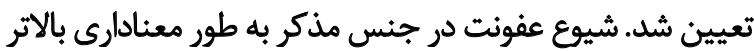

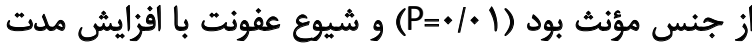

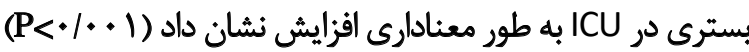

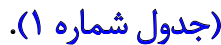

شايعترين عفونت در سالمندان مورد مطالعه، عفونت تنفسى ارئ

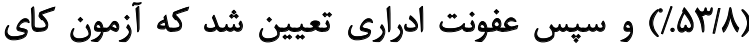

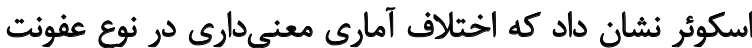

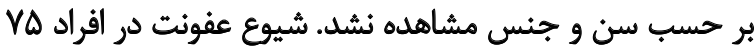

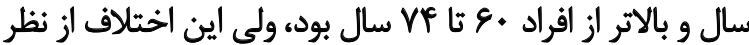

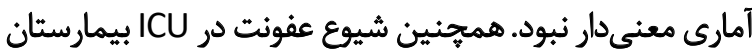

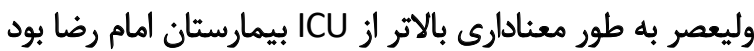

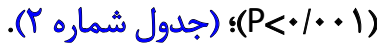

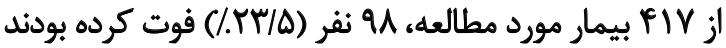

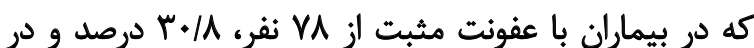

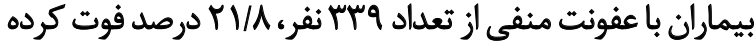

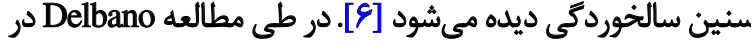

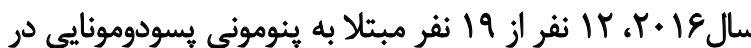

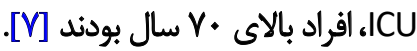

در افراد سالمند، علاوه بر احتمال ابتلاى بيشتر به بيمارىهاى

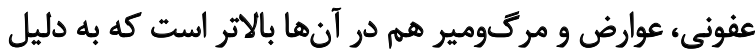

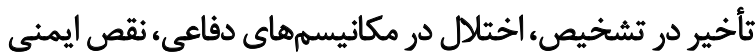

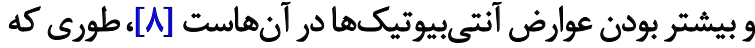

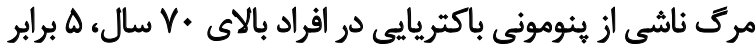

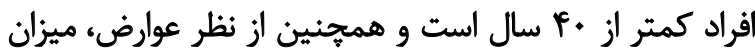

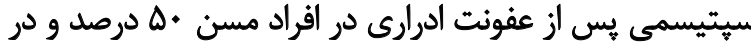

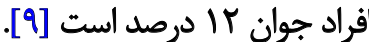

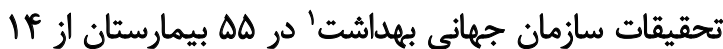

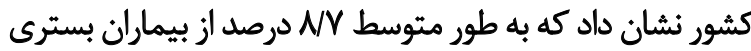

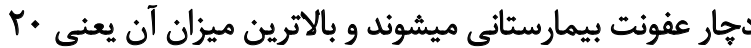

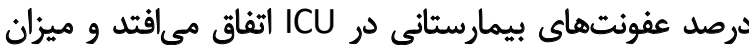

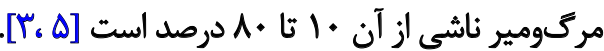
هدف ازز انجام اين مطالعه، بررسى شيوع وعوامل خطر عفونتها

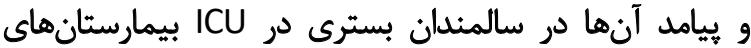

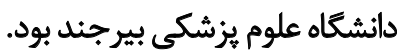

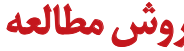

در اين مطالعه مقطعى (توصيفى-تحليلى) جمعيت مورد

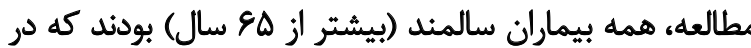

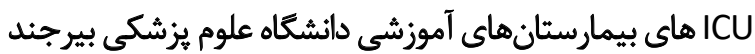

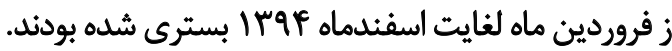

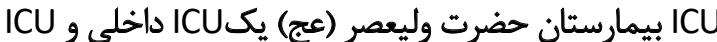

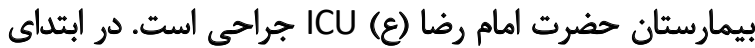

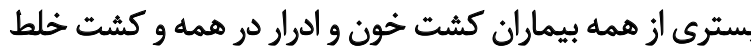
و ترشح زخم در صورت داشتن نمونه انجام شد.

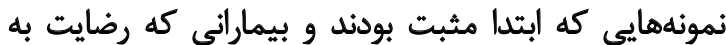

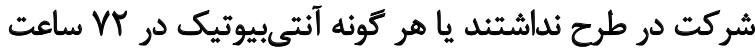

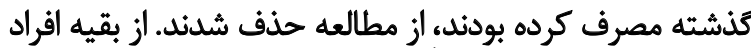

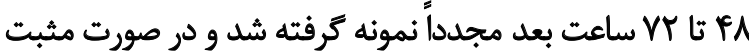

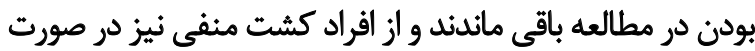

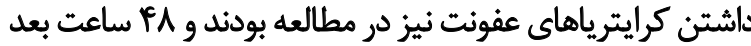
هم كشت كرفته شد.

نمونهكيرى را سويروايزر آموزشديده ICU ها با نظرات

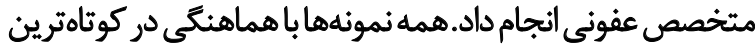

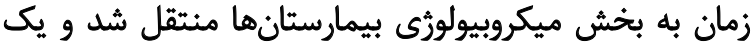

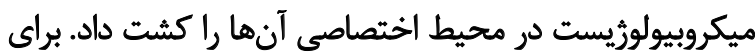


جدول ا. مقايسه شيوع عفونت در بيماران بسترى در بخشهاى UCI برحسب جنس و مدت بسترى در بيمارستان

\begin{tabular}{|c|c|c|c|c|}
\hline \multirow{2}{*}{ 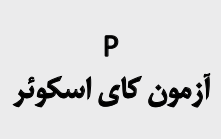 } & \multicolumn{2}{|c|}{ فراواتي (درصد) } & \multirow[t]{2}{*}{ عفونت } & \multirow[b]{2}{*}{ 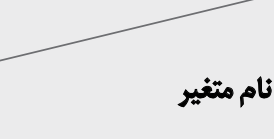 } \\
\hline & ندارد & مارد & & \\
\hline \multirow{2}{*}{$\mathrm{P}=* 1+1$} & $I F \Delta(q / V \Delta)$ & $F \&(M \in / I)$ & مرد & \multirow{2}{*}{ جنس } \\
\hline & $\operatorname{l9F}(\Lambda ৯ / \Lambda)$ & $M(N F / T)$ & نن & \\
\hline \multirow{3}{*}{$P<+1++1$} & $\operatorname{r\Delta V}(৭ V / r \Delta)$ & $V(T / M)$ & S ه روز & \multirow{3}{*}{ مدت بسترى در بيمارستان } \\
\hline & g. (qq/A) & $r \varepsilon(\Gamma+/)$ & . ا-ه روز & \\
\hline & $M T(T r / A)$ & $f \Delta(\mathscr{V V} / T)$ & i. 1.2 & \\
\hline
\end{tabular}

جدول شماره r. مقايسه شيوع عفونت در بيماران بسترى در بخش هاى UCl بر حسب سن و بيمارستان مورد مطالعه

\begin{tabular}{|c|c|c|c|c|}
\hline \multirow{2}{*}{ 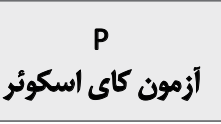 } & \multicolumn{2}{|c|}{ فراواتى (درصد) } & \multirow[t]{2}{*}{ عفونت } & \multirow[b]{2}{*}{ نام متغير } \\
\hline & ندارد و مثفى & دارد و مثبت & & \\
\hline \multirow{2}{*}{$\mathrm{P}=\cdot / p q$} & In (AT/P) & $r e(\mid Y / F)$ & (ال & \multirow[b]{2}{*}{ سن } \\
\hline & $\lg \Lambda(\Lambda \cdot)$ & $P(T \cdot)$ & Vه Vال و بالاتر & \\
\hline \multirow{2}{*}{$\mathrm{P}<+1 .+1$} & $T T \Delta(a T / T)$ & $r \cdot(V / A)$ & امام رضا ICU & \multirow{2}{*}{ نام بيمارستان } \\
\hline & $1 . f(R / T)$ & $\Delta A(r \Delta / A)$ & وليعصر ICU & \\
\hline
\end{tabular}

il

جدول شماره r. مقايسه ميزان مركومير بر حسب وضعيت عفوئت در بيماران مورد مطالعه

\begin{tabular}{|c|c|c|c|}
\hline & & & \\
\hline ندارد (منفى) & دارد (مثبت) & & صعيت مرى \\
\hline$V \in(T / A)$ & $r(r \cdot / \Lambda)$ & فوتشده & \\
\hline rEA (NNT) & $\Delta P(g Q / T)$ & فوتنشده & \\
\hline$m q(1 .)$. & $v \wedge(1 \cdot)$. & جمع & \\
\hline
\end{tabular}

证

مطالعات مختلف نشان دادند كه عفونتهاى بيمارستانى در

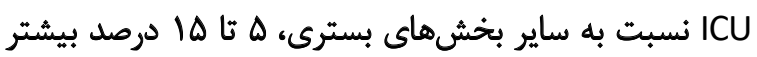

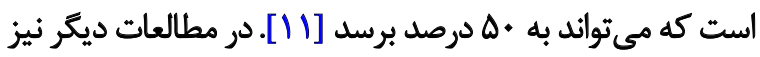

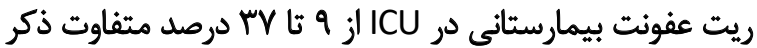

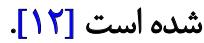

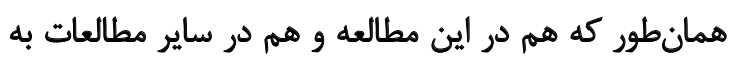

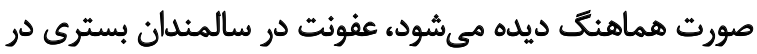

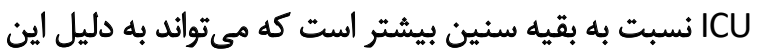

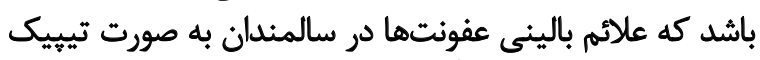

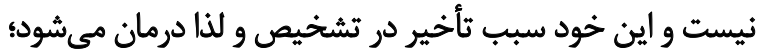

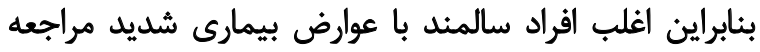

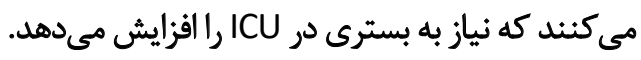

بودند كه اين اختلاف با استفاده از آزمون كاى اسكوئر از نظر

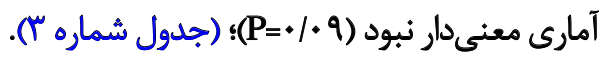
بحث

در اين مطالعه كه هدف آن بررسى عفونتها و بيامد آنها در

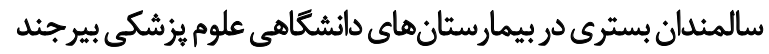

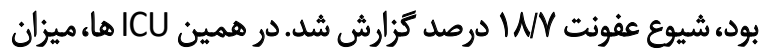

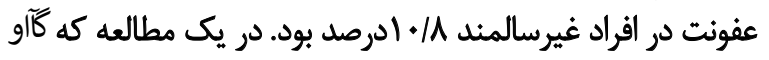

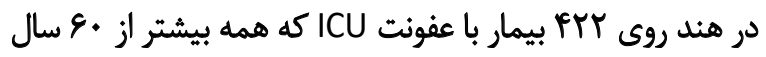

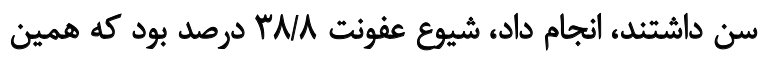

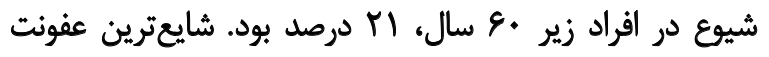

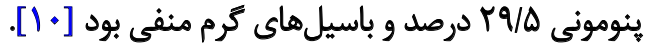


علاوه بر عفونت ناشى از لوله كذارى، ينومونى آسييراسيون و ينومونى بيمارستائى درافرادسالمندر ابايدبها آنها اضافه كردي.

در اين مطالعه از نظر علاثم بيمارىزا شايعترين ارخانيسم در عفونت

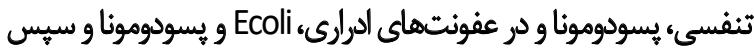

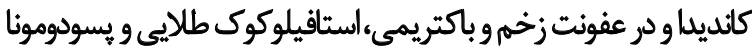

بونئد.

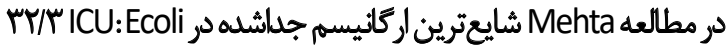

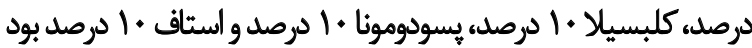

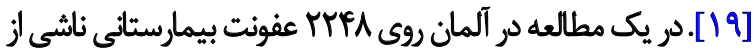
INF، ICU

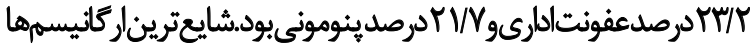

M Ecoli

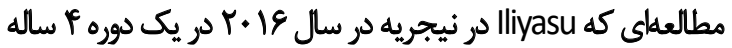

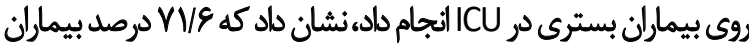

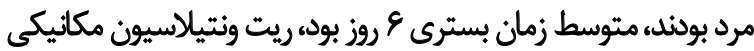

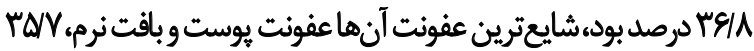

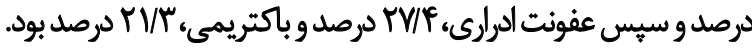

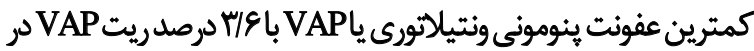

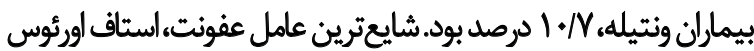

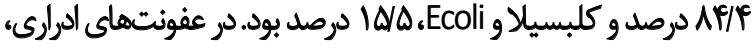

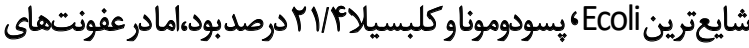

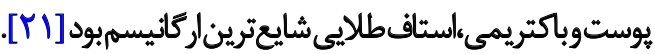

همانطوركه مشهود است انتروباكترياسه شامل Ecoli و كلبسيلاو

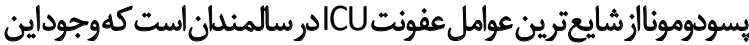

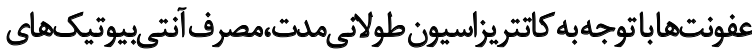

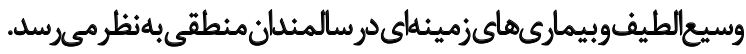

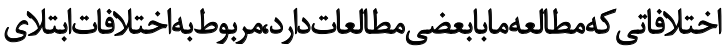

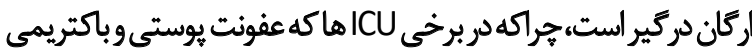

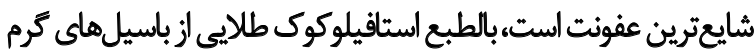

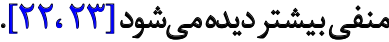

در مطالعهاميزان شيوع عفونتهادر ابيمارستان حضرت توليعصر

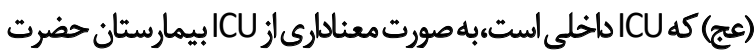

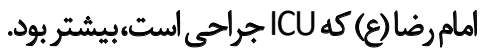

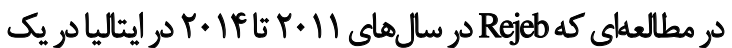

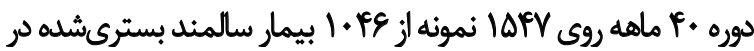

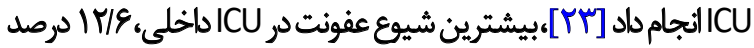

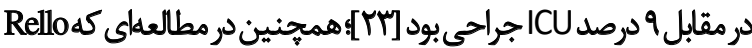

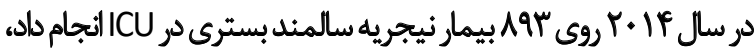

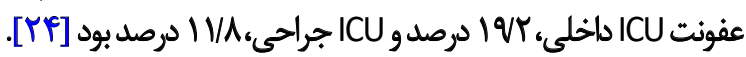
همهسو بودن مطالعه ما با مطالعات ديكر در رابطه با افزايش عفونت در

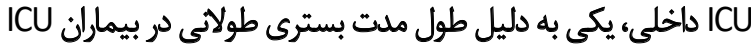

ازطرفي نقصايمنى سلولاركهبهصورتفيزيولوزيكيكدر سالمنداناتفات

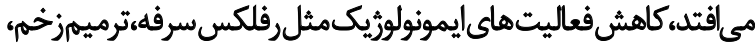

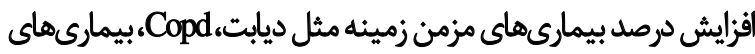

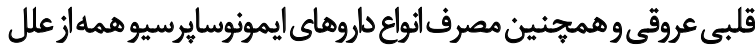

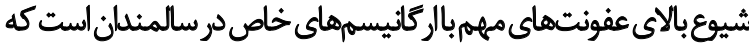

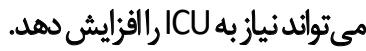
در مطالعه ماءميزان عفونت باطول ملت بسترى وجنس مذكر ارتباط

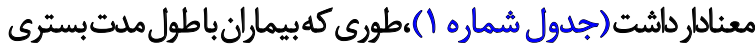

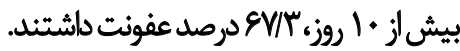

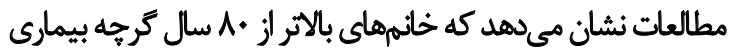

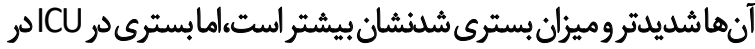

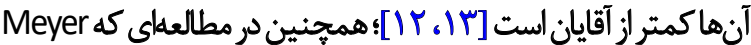

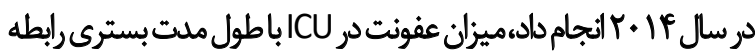

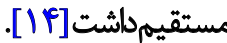

افزايش طول ملت بسترى در ICU هم به خوديى خود و هم به واسطة

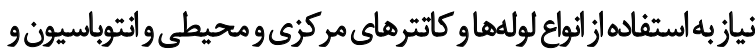

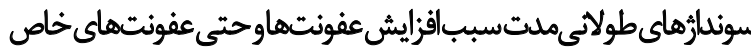

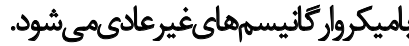

شايعترين عفونت در مطالعه ما،عفونت تنفسى / مآه درصدو سيس

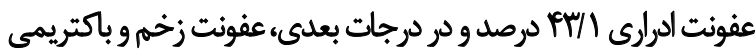

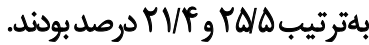

در مطالعه Mithrawal هم شايعترين عفونت ICU در سالمندان،

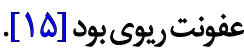

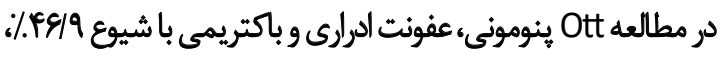

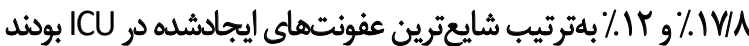

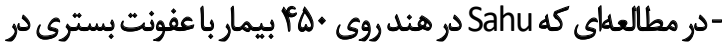

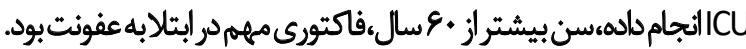

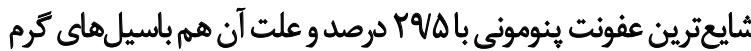

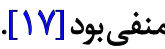
تشايعترين محل عفونت، ادرارى \$ \$ درصد، ريوى IT/Q I درصد،

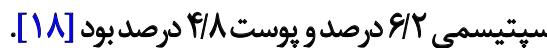
انتروباكترياسه، شايعترين اركانيسمهالى عفونت بيمارستانى وعفونت

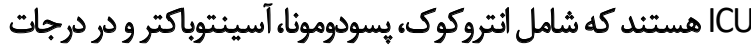

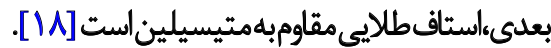
شيوع بالاى عفونت تنفسى در مطالعه ما و اغلب مطالعات به دليل

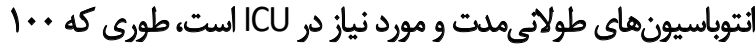

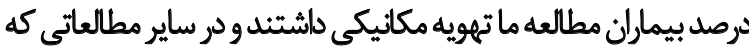

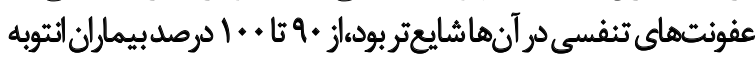
. 
را تشكيل مي دهند و تظاهرات عفونت و بيمارىهاى آنها تيييك

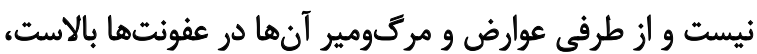

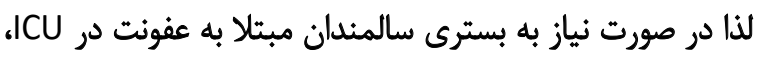

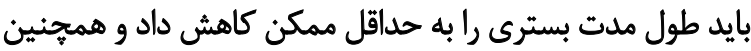

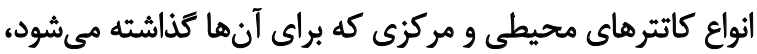

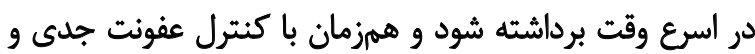

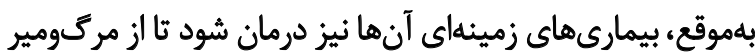

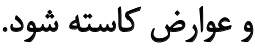

\section{ماحظاتازخلاقي

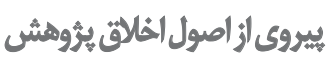

اينمطالعهبخشى اززطرحتحقيقاتى باعنوان إيياميولورىبيمارىهاى

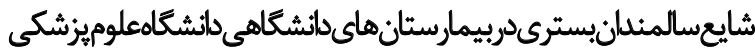

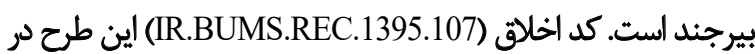

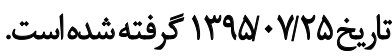

$$
\text { مامي مالى }
$$

اين طرح با حمايت مالى معاونت تحقيقات و فناورى دانشكاه علوم يزشكى بير جند انجام شده است.

$$
\text { مشاركت نويسند مكان }
$$

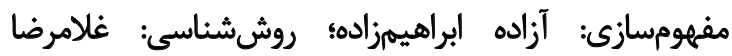

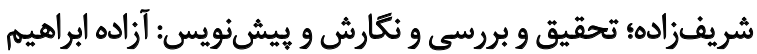

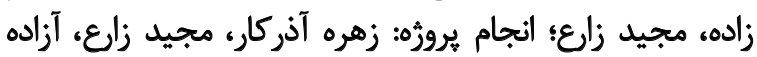

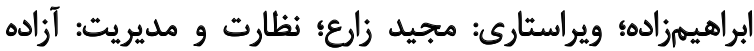

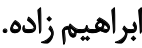

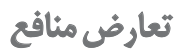

وجنابر اظهار نويسند كان، هيج كونه تعارض منافع مادى و معنوى

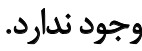

$$
\text { تشكو و قدردانى }
$$

بدينوسيله از زحمات سويروايزرهاي محترم ICU بيمارستان حضرت وليعصر (عج) و بيمارستان امام رضا (ع)، كارشناس إناس

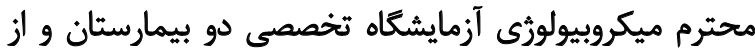
بيماران مورد مطالعه تشكر و قدردانى مي شئود.

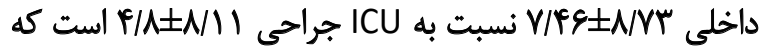

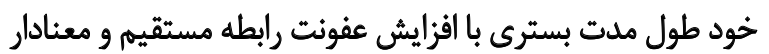

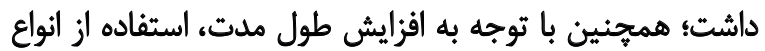

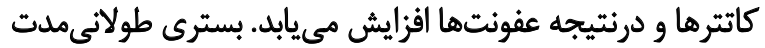

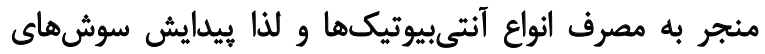

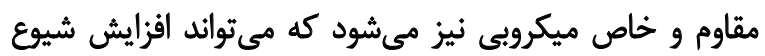
عفونت را در ICU داخلى را توجيه كند.

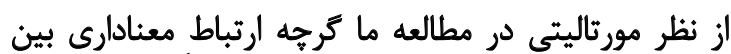

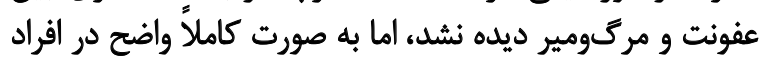

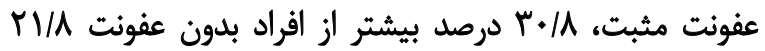

در صل يود.

مورتاليتى در كروهى كه با عفونت مثبت فوت شدند، شايعترين

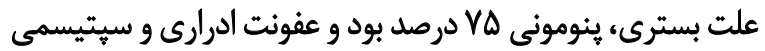

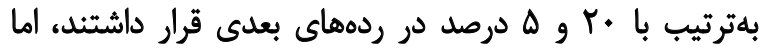

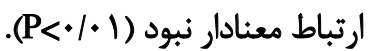
كونومونى ها با توجه به اركانيسم عامل بيمارى كه اغلب

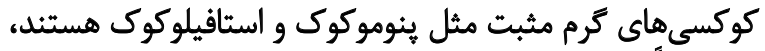

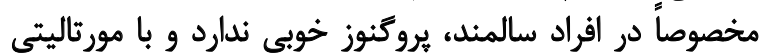

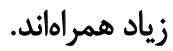

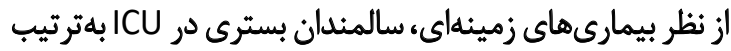

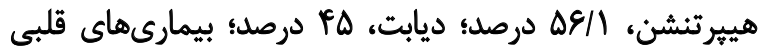

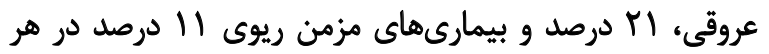
كروه با مورتاليتى و بدون مورتاليتى بودندي.

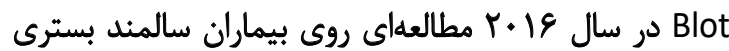

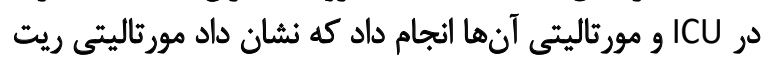

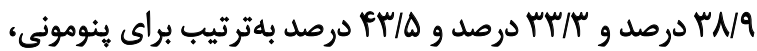

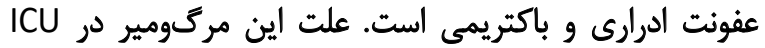

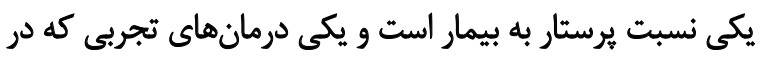

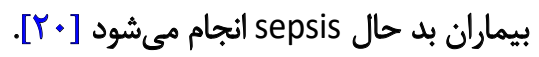
در مطالعه Iregbu كه روى سالمندان بسترى در icu انجام داد،

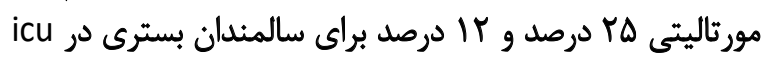

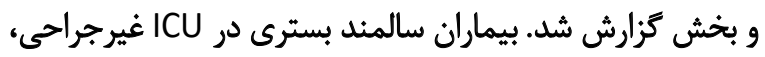

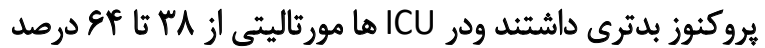

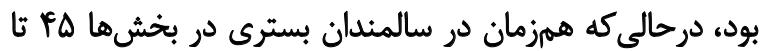

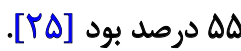
كاملاً منطقى است كه در سالمندان با وجود بيمارىهاى بودي

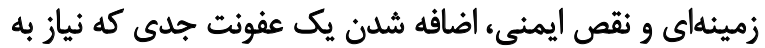

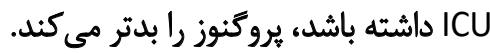
نتيجه كيرى نهبايى با توجه به اينكه سالمندان درصد نسبثاً زيادى از افراد جامعه 


\section{References}

[1] Zilahi G, Artigas A, Martin-Loeches I. What's new in multidrugresistant pathogens in the ICU? Annals of Intensive Care. 2016; 6(1):96. [DOI:10.1186/s13613-016-0199-4] [PMID] [PMCID]

[2] Flaatten H, De Lange DW, Morandi A, Andersen FH, Artigas A, Bertolini G, et al. The impact of frailty on ICU and 30-day mortality and the level of care in very elderly patients ( $\geq 80$ years). Intensive Care Medicine. 2017; 43(12):1820-8. [DOI:10.1007/s00134017-4940-8] [PMID]

[3] Pintado MC, Villa P, González-García N, Luján J, Molina R, Trascasa M, et al. Characteristics and outcomes of elderly patients refused to ICU. The Scientific World Journal. 2013; 2013. [DOI:10.1155/2013/590837] [PMID] [PMCID]

[4] Sprung CL, Artigas A, Kesecioglu J, Pezzi A, Wiis J, Pirracchio R, et al. The Eldicus prospective, observational study of triage decision making in European intensive care units. Part II: intensive care benefit for the elderly. Critical Care Medicine. 2012; 40(1):1328. [DOI:10.1097/CCM.0b013e318232d6b0] [PMID]

[5] Blot S, Koulenti D, Dimopoulos G, Martin C, Komnos A, Krueger WA, et al. Prevalence, risk factors, and mortality for ventilator-associated pneumonia in middle-aged, old, and very old critically ill patients. Critical Care Medicine. 2014; 42(3):601-9. [DOI:10.1097/01.ccm.0000435665.07446.50] [PMID]

[6] Behnke M, Hansen S, Leistner R, Diaz LAP, Gropmann A, Sohr $\mathrm{D}$, et al. Nosocomial infection and antibiotic use: a second national prevalence study in Germany. Deutsches Ärzteblatt International. 2013; 110(38):627. [DOI:10.3238/arztebl.2013.0627] [PMID] [PMCID]

[7] Del Bono V, Giacobbe DR. Bloodstream infections in internal medicine. Virulence. 2016; 7(3):353-65. [DOI:10.1080/21505594.20 16.1140296] [PMID] [PMCID]

[8] Ellison J, Southern D, Holton D, Henderson E, Wallace J, Faris $\mathrm{P}$, et al. Hospital ward design and prevention of hospitalacquired infections: A prospective clinical trial. Canadian Journal of Infectious Diseases and Medical Microbiology. 2014; 25. [DOI:10.1155/2014/685402] [PMID] [PMCID]

[9] Gao J, Ying K. Homology Analysis and Nosocomial Infection Control on Outbreak of ICU Acinetobacter baumannii Pneumonia. Chest. 2016; 149(4):A93. [DOI:10.1016/j.chest.2016.02.098]

[10] Solis-Hernandez PS, Vidales-Reyes M, Garza-Gonzalez E, Guajardo-Alvarez G, Chavez-Moreno S, Camacho-Ortiz A. Hospitalacquired infections in elderly versus younger patients in an acute care hospital.International Journal of Infection. 2016; 3(1):e32620. [DOI:10.17795/iji-32620]

[11] Le NK, Wertheim H, Vu PD, Khu DTK, Le HT, Hoang BTN, et al. High prevalence of hospital-acquired infections caused by gram-negative carbapenem resistant strains in Vietnamese pediatric ICUs: A multi-centre point prevalence survey. Medicine. 2016; 95(27): e4099. [DOI:10.1097/MD.0000000000004099] [PMID] [PMCID]

[12] Luzzati R, Merelli M, Ansaldi F, Rosin C, Azzini A, Cavinato $\mathrm{S}$, et al. Nosocomial candidemia in patients admitted to medicine wards compared to other wards: a multicentre study. Infection. 2016; 44(6):747-55. [DOI:10.1007/s15010-016-0924-9] [PMID]

[13] Mancini A, Verdini D, La Vigna G, Recanatini C, Lombardi FE, Barocci S. Retrospective analysis of nosocomial infections in an Italian tertiary care hospital. New Microbiol. 2016; 39(3):197-205.
[14] Meyer E, Schröder C, Gastmeier P, Geffers C. The reduction of nosocomial MRSA infection in Germany: an analysis of data from the Hospital Infection Surveillance System (KISS) between 2007 and 2012. Deutsches Ärzteblatt International. 2014; 111(19):331-6. [DOI:10.3238/arztebl.2014.0331] [PMID] [PMCID]

[15] Mitharwal SM, Yaddanapudi S, Bhardwaj N, Gautam V, Biswal M, Yaddanapudi L. Intensive care unit-acquired infections in a tertiary care hospital: An epidemiologic survey and influence on patient outcomes. American Journal of Infection Control. 2016; 44(7):e113-e7. [DOI:10.1016/j.ajic.2016.01.021] [PMID]

[16] Ott E, Saathoff S, Graf K, Schwab F, Chaberny IF. The prevalence of nosocomial and community acquired infections in a university hospital: an observational study. Deutsches Ärzteblatt International. 2013; 110(31-32):533-40. [DOI:10.3238/arztebl.2013.0533] [PMID] [PMCID]

[17] Sahu MK, Siddharth B, Choudhury A, Vishnubhatla S, Singh $\mathrm{SP}$, Menon R, et al. Incidence, microbiological profile of nosocomial infections, and their antibiotic resistance patterns in a high volume Cardiac Surgical Intensive Care Unit. Annals of Cardiac Anaesthesia. 2016; 19(2):281. [DOI:10.4103/0971-9784.179625] [PMID] [PMCID]

[18] Custovic A, Smajlovic J, Hadzic S, Ahmetagic S, Tihic N, Hadzagic H. Epidemiological surveillance of bacterial nosocomial infections in the surgical intensive care unit. Materia Socio-medica. 2014; 26(1):7-11. [DOI:10.5455/msm.2014.26.7-11] [PMID] [PMCID]

[19] Mehta Y, Gupta A, Todi S, Myatra S, Samaddar D, Patil V, et al. Guidelines for prevention of hospital acquired infections. Indian Journal of Critical Care Medicine: Peer-reviewed, Official Publication of Indian Society of Critical Care Medicine. 2014; 18(3):149-63. [DOI:10.4103/0972-5229.128705] [PMID] [PMCID]

[20] Moreira MR, Guimarães MP, Rodrigues AAdA, Gontijo Filho PP. Antimicrobial use, incidence, etiology and resistance patterns in bacteria causing ventilator-associated pneumonia in a clinical-surgical intensive care unit. Revista da Sociedade Brasileira de Medicina Tropical. 2013; 46(1):39-44. [DOI:10.1590/0037868216722013] [PMID]

[21] Iliyasu G, Daiyab FM, Tiamiyu AB, Abubakar S, Habib ZG, Sarki AM, et al. Nosocomial infections and resistance pattern of common bacterial isolates in an intensive care unit of a tertiary hospital in Nigeria: A 4-year review. Journal of Critical Care. 2016; 34:116-20. [DOI:10.1016/j.jcrc.2016.04.018] [PMID]

[22] Iregbu K, Sonibare S. Profile of infections in Intensive Care Unit (ICU) in a central Nigeria tertiary hospital. African Journal of Clinical and Experimental Microbiology. 2015; 16(1):23-7. [DOI:10.4314/ajcem.v16i1.4]

[23] Ben Rejeb M, Sahli J, Chebil D, Khefacha-Aissa S, Jaidane N, Kacem B, et al. Mortality among Patients with Nosocomial Infections in Tertiary Intensive Care Units of Sahloul Hospital, Sousse, Tunisia. Archives of Iranian Medicine. 2016; 19(3):84960119025.

[24] Rello J, Lisboa T, Koulenti D. Respiratory infections in patients undergoing mechanical ventilation. The Lancet Respiratory Medicine. 2014; 2(9):764-74. [DOI:10.1016/S2213-2600(14)70171-7]

[25] Vosylius S, Sipylaite J, Ivaskevicius J. Determinants of outcome in elderly patients admitted to the intensive care unit. Age and Ageing. 2005; 34(2):157-62. [DOI:10.1093/ageing/afi037] [PMID] 
This Page Intentionally Left Blank 